\title{
FURTHER REMARKS ON ASYMPTOTIC BEHAVIOR OF THE NUMERICAL SOLUTIONS OF PARABOLIC BLOW-UP PROBLEMS*
}

\author{
CHIEN-HONG $\mathrm{CHO}^{\dagger}$ AND HISASHI OKAMOTO
}

\begin{abstract}
We consider a semilinear parabolic equation $u_{t}=u_{x x}+f(u)(0<x<1,0<t)$, and a finite difference approximation for it. We discuss the way how the asymptotic profile of the blow-up solution is reproduced by the numerical solution. We will also determine qualitatively the influence of the definition of time mesh on the blow-up set of the numerical solution. Moreover, we show that explicit and implicit schemes may claim different blow-up sets.
\end{abstract}

Key words. Finite difference method, nonlinear parabolic equation, blow-up.

AMS subject classifications. $65 \mathrm{M} 06$

1. Introduction. We consider the following semilinear parabolic equation

$$
u_{t}(t, x)=u_{x x}(t, x)+f(u(t, x)) \quad(0<x<1,0<t),
$$

where the subscripts denote differentiation, with the initial and boundary conditions:

$$
u(0, x)=u_{0}(x) \quad(0<x<1), \quad u(t, 0)=u(t, 1)=0 \quad(0<t) .
$$

It is known (see $[8,10,11])$ that a solution with a large initial data blows up in finite time under certain growth assumptions on $f$ as $u \rightarrow \infty$. Researches on parabolic blow-up problems like the present one have made considerable progress, and detailed knowledge on asymptotic profiles near the blow-up time, blow-up rate, complete and/or incomplete blow-up etc. have been established. See, for instance, [8], [9] or $[15]$.

Compared with the theoretical study, numerical analysis of the blow-up problem does not seem to be explored enough. Our purpose in the present paper is to provide some mathematical analysis on finite difference approximations. Let us briefly recall some investigations of the past. Nakagawa[13] considered an explicit finite difference scheme with uniform spatial grids and adaptive step sizes in time. He showed that his numerical solutions converge to the solution up to the blow-up time. This implies not only that the numerical solutions converge in the time interval where the solution is smooth but also that the numerical blow-up time converges to the real blow-up time. If we recall that the convergence, in usual numerical analysis, is proved under some smoothness assumptions, this result may be remarkable, since regularity of the solution is lost at the blow-up time. His result was later improved substantially by $[1,2,3]$. Later, Chen[5] considered a similar problem and showed that the "blow-up set" of the numerical solution can be different from the blow-up set of the solutions of PDE. The problems which were left unanswered in these papers were dealt with in $[6,7]$, and many of them were solved. However, some questions were left in [7] as open questions, and we would like to shed light on these questions in the present paper.

The rest of the paper is organized as follows. In section 2, known results are recalled and problems to be addressed in the present paper will be explained. In

\footnotetext{
*Received December 26, 2007; accepted for publication May 9, 2008.

${ }^{\dagger}$ Research Institute for Mathematical Sciences, Kyoto University, Kyoto 606-8502, Japan. Supported by Studying Abroad Scholarship (Taiwan, R.O.C.).

${ }^{\ddagger}$ Research Institute for Mathematical Sciences, Kyoto University, Kyoto 606-8502, Japan (okamoto@kurims.kyoto-u.ac.jp). Partially supported by JSPS Grant-in-Aid 17204008.
} 
section 3, we consider the influence of the definition of time increments on the blowup set of numerical solution. The difference between explicit and implicit schemes are revealed. In section 4 , the blow-up rate for the numerical solution will be derived when $f(u)=u[\log (1+u)]^{\beta} \quad(\beta>1)$ and some numerical experiments in this case will also be reported here.

2. Some background materials and the problems to be considered. We here recall results of [7]. Let $N$ be a positive integer, and consider the following difference scheme.

$$
\frac{u_{j}^{n+1}-u_{j}^{n}}{\triangle t_{n}}=\frac{u_{j+1}^{n}-2 u_{j}^{n}+u_{j-1}^{n}}{h^{2}}+f\left(u_{j}^{n}\right) \quad\left(\begin{array}{l}
j=1, \cdots, N-1 \\
n=0,1, \cdots
\end{array}\right) .
$$

Here, $u_{j}^{n}$ is an approximation for $u\left(t_{n}, x_{j}\right) . x_{j}=j / N(j=0,1, \cdots, N)$ are the grid points, and $N h=1$. We set $u_{0}^{n}=u_{N}^{n}=0$ as the boundary condition. The initial condition is set as $u_{j}^{0}=u_{0}\left(x_{j}\right)$. $t_{n}$ denotes the $n$-th discrete time step, which is defined by $t_{0}=0$ and

$$
t_{n}=t_{n-1}+\triangle t_{n-1}=\sum_{k=0}^{n-1} \triangle t_{k}, \quad \text { for } n>0
$$

where $\triangle t_{k}=t_{k+1}-t_{k}$ denotes the discrete time increment, defined as

$$
\triangle t_{k}=\tau \cdot \min \left\{1, \frac{1}{H\left(\left\|u^{k}\right\|_{p}\right)}\right\} .
$$

Here $\tau>0$ is a prescribed parameter, $H$ is a prescribed function, and $\left\|u^{k}\right\|_{p}$ denotes the discrete $L^{p}$ norm, namely,

$$
\left\|u^{k}\right\|_{p}= \begin{cases}\left(h \sum_{j=1}^{N-1}\left(u_{j}^{k}\right)^{p}\right)^{1 / p} & (1 \leq p<\infty), \\ \max _{0 \leq j \leq N} u_{j}^{k} & (p=\infty) .\end{cases}
$$

(In the present paper, we consider only non-negative initial data, whence all the unknowns are non-negative.)

DeFinition 2.1. We define

$$
T(\tau, h)=\lim _{n \rightarrow \infty} t_{n}\left(\text { which is equal to } \sum_{k=0}^{\infty} \triangle t_{k}\right)
$$

and call it the numerical blow-up time.

DeFinition 2.2. The set

$$
\left\{x_{j} \mid \lim _{n \rightarrow \infty} u_{j}^{n}=\infty\right\}
$$

is called the numerical blow-up set.

As for the nonlinearity $f$, we assume the same assumption as in [7]. Namely, 
i) $f$ is continuously differentiable;

ii) $f(s)>0$ for $s>0$;

iii) $f$ is strictly monotone increasing and convex in $0<s<\infty$;

iv) $\int_{1}^{\infty} \frac{\mathrm{d} s}{f(s)}<\infty$.

With this setting, the following theorem was proved in [7]:

THEOREM 2.1. Let $\tau / h^{2}<1 / 2$. Assume that $H(s)$ satisfies that $H(s) \rightarrow \infty$ as $s \rightarrow \infty$, that the mapping $s \longmapsto s+\tau \frac{f(s)}{H(s)}$ is monotone increasing in $H^{-1}(1) \leq s<\infty$ and that $\int_{1}^{\infty} \frac{f^{\prime}(s)}{f(s) H(s)} \mathrm{d} s<\infty$. Suppose that $u_{0}$ satisfies the conditions (A0) and (A3) below, and let $T$ be the blow-up time of (1). Let $T^{\prime} \in(0, T)$ be chosen arbitrarily. Then the solution of (3) converges to the solution of (1) as far as $t_{n} \leq T^{\prime}$. Furthermore, the numerical blow-up time is finite: $T(\tau, h)=\sum_{n=0}^{\infty} \triangle t_{n}<\infty$. Moreover, $T(\tau, h)$ converges to $T$ as $h \rightarrow 0$.

The reader should note that quite a large class of functions can be used for $H$. For instance, if $f(u)=u^{1+\alpha}$ with $\alpha>0$, then $H(s)=s^{\gamma}$ with any $0<\gamma<1+\alpha+\tau^{-1}$ satisfies the assumptions of the theorem. Also, any $p$ can be used. Some people use $H(s)=s^{\alpha}$ when $f(u)=u^{1+\alpha}$, but no conclusive evidence for the superiority of this choice has been established. $p=\infty$ is a convenient choice, but we do not know which $p$ is best from numerical analytic viewpoint. More researches are needed in this direction. The question on the rate of the convergence $T(\tau, h) \rightarrow T$ is also unknown. It depends on $H$ and $p$, but we are unable to determine the dependence.

We next consider the blow-up set. Throughout the rest of the paper, we assume that the initial function satisfies the following conditions:

(A0) $u_{0}$ is continuous and non-negative in $0 \leq x \leq 1 . u_{0}(0)=u_{0}(1)=0$, but $u_{0} \not \equiv 0$;

(A1) $u_{0}$ is spatially symmetric about $x=1 / 2$;

(A2) $u_{0}$ is strictly monotone increasing in $[0,1 / 2]$;

(A3) $u_{0}$ is large in the sense that $\int_{0}^{1} u_{0}(x) \sin x \mathrm{~d} x>c_{0}$, where $c_{0}$ is a certain constant depending only on $f$ (see Lemma 3.2 of [7]).

Under these conditions, it is known that the solution of (1) and (2) blows up only at the central point; that is,

$$
\lim _{t \rightarrow T} u\left(t, \frac{1}{2}\right)=\infty, \quad \limsup _{t \rightarrow T} u(t, x)<\infty \quad(x \neq 1 / 2),
$$

where $T$ denotes the blow-up time. This phenomenon, called the single-point blowup, was first proved by Weissler [14] under a more restrictive condition on the initial data. The general result is due to Friedman and McLeod [10]. See also [4].

Chen [5] considered the finite difference scheme (3) when $f(u)=u^{1+\alpha}, H(s)=s^{\alpha}$ and $p=2$. He assumed that $N$ is even, say $N=2 m$, so that $x=1 / 2$ is one of the grid points. He proved that if $\alpha>1$, then

$$
\lim _{n \rightarrow \infty} u_{m}^{n}=\infty, \quad \limsup _{n \rightarrow \infty} u_{j}^{n}<\infty \quad(j \neq m)
$$


and that if $\alpha \leq 1$, then

$$
\lim _{n \rightarrow \infty} u_{m}^{n}=\infty, \quad \lim _{n \rightarrow \infty} u_{m \pm 1}^{n}=\infty .
$$

Since we already know for any $\alpha>0$ that only $x=1 / 2=x_{m}$ is the blow-up point for the solution of (1), his result shows an interesting discrepancy between the continuous and discrete problems. He also proved that

$$
\limsup _{n \rightarrow \infty} u_{j}^{n}<\infty
$$

for all $j \neq m, m \pm 1$ if $\alpha=1$. But he could not determine the number of the blow-up set in the case of $\alpha<1$. Later, [7] determined precisely the number of blow-up set as a function of $\alpha \in(0,1)$.

In the present paper we consider the case where $H(s)=s^{\gamma}$, where $\gamma<\alpha$, and we will show that $\gamma$ changes the blow-up profile considerably.

More pathological phenomena occur if the nonlinearity $f$ is a milder one, such as $f(u)=u[\log (1+u)]^{\beta}$ with $\beta>1$. Lacey[12] showed that the solution of (1) and (2) with $f(u)=u[\log (1+u)]^{\beta}$ blows up at exactly one point if $\beta>2$ and blows up at all points in $(0,1)$ if $1<\beta<2$, while regional blow-up occurs, that is, the solution blows up at a proper subset of $(0,1)$ with positive measure for $\beta=2$. The authors[7] considered a convergent numerical scheme and found that the numerical solution blows up at all the grid points whatever the value of $\beta$ may be. The result is rather interesting, since single-point blow-up in the case of $\beta>2$ cannot be reproduced, no matter how small the spatial grid size may be.

In section 4, we will show a different criterion of blow-up, by which we can reproduce the single-point blow-up in the case of $\beta>2$.

3. Definition of $\triangle t_{n}$ and its influence on blow-up set. In this section, we assume that $f(u)=u^{2}$. The results in $[5,7]$ tell us that

$$
\lim _{n \rightarrow \infty} u_{m}^{n}=\infty, \quad \lim _{n \rightarrow \infty} u_{m \pm 1}^{n}=\infty, \quad \limsup _{n \rightarrow \infty} u_{j}^{n}<\infty \quad(j \neq m, m \pm 1),
$$

if we define $\triangle t_{n}$ by

$$
\triangle t_{n}=\tau \cdot \min \left\{1, \frac{1}{\left\|u^{n}\right\|_{\infty}}\right\} .
$$

We now consider the case where

$$
\triangle t_{n}=\tau \cdot \min \left\{1, \frac{1}{\left\|u^{n}\right\|_{\infty}^{\gamma}}\right\}
$$

with $\gamma<1$, which obviously satisfies the assumptions in Theorem 2.1. By the symmetry and positivity of the solution, the definition of the time increments becomes

$$
\triangle t_{n}=\tau \cdot \min \left\{1, \frac{1}{\left(u_{m}^{n}\right)^{\gamma}}\right\} .
$$

The result of the present section is roughly summarized as follows: The smaller $\gamma$ is, the more blow-up points appear. Note that, by Theorem 2.1, the numerical blowup time converges for all $\gamma \in(0,2]$. Theorem 2.1 cannot decide which $\gamma$ is the best. However, $\triangle t_{n}$ becomes small for a large $\gamma$. In order to compute fast, a large $\triangle t_{n}$ is preferable, and it is better to take a smaller $\gamma$. As far as the convergence of $T(\tau, h)$ is concerned, this is fine. But the theorems below show that the blow-up profile is difficult to reproduce for a smaller $\gamma$. 
3.1. Explicit scheme. We consider in this subsection the following explicit scheme: the implicit scheme will be analyzed in the next subsection.

$$
\frac{u_{j}^{n+1}-u_{j}^{n}}{\triangle t_{n}}=\frac{u_{j+1}^{n}-2 u_{j}^{n}+u_{j-1}^{n}}{h^{2}}+\left(u_{j}^{n}\right)^{2} \quad\left(\begin{array}{l}
j=1, \cdots, N-1 \\
n=0,1, \cdots
\end{array}\right),
$$

where $\triangle t_{n}$ is given by (5). Throughout this subsection we assume that $\tau / h^{2}<1 / 2$. Since we assume (A0)-(A3), it holds that $0=u_{0}^{n}<u_{1}^{n}<\cdots<u_{m-1}^{n}<u_{m}^{n}$ for all $n$ and that

$$
\lim _{n \rightarrow \infty} u_{m}^{n}=\infty .
$$

(Recall that $N=2 m$ and $x_{m}=1 / 2$.)

TheOrem 3.1. Let (A0)-(A3) be assumed and let $\left\{u_{j}^{n}\right\}$ be the solution of (6) with (5). Then for all $0<\gamma \leq 1$, we have

$$
\lim _{n \rightarrow \infty} u_{m-1}^{n}=\infty .
$$

If $0<\gamma \leq(3-\sqrt{5}) / 2$, then

$$
\lim _{n \rightarrow \infty} u_{m-2}^{n}=\infty
$$

Proof. The equation (7) was proved by $[5,7]$ in the case of $\gamma=1$. The proof is actually easier in the case of $\gamma<1$. Let $\lambda=\tau / h^{2}$ and $\lambda_{n}=\Delta t_{n} / h^{2}$. Then the equation (6) can be written, for $j=m-1$, as

$$
u_{m-1}^{n+1}=\left(1-2 \lambda_{n}\right) u_{m-1}^{n}+\lambda_{n} u_{m}^{n}+\lambda_{n} u_{m-2}^{n}+\Delta t_{n}\left(u_{m-1}^{n}\right)^{2} .
$$

Since every term is positive, and since $\Delta t_{n}=\tau\left(u_{m}^{n}\right)^{-\gamma}$ for sufficiently large $n$, we have

$$
u_{m-1}^{n+1} \geq \lambda_{n} u_{m}^{n}=\lambda\left(u_{m}^{n}\right)^{1-\gamma} \rightarrow \infty \quad(\text { as } n \rightarrow \infty) .
$$

We next prove that

$$
\lim _{n \rightarrow \infty} \frac{u_{m}^{n+1}}{\left(u_{m}^{n}\right)^{2-\gamma}}=\tau
$$

To prove this, note that

$$
\begin{aligned}
u_{m}^{n+1} & =u_{m}^{n}+2 \lambda_{n}\left(u_{m-1}^{n}-u_{m}^{n}\right)+\triangle t_{n}\left(u_{m}^{n}\right)^{2} \\
& =u_{m}^{n}+2 \lambda_{n}\left(u_{m-1}^{n}-u_{m}^{n}\right)+\tau\left(u_{m}^{n}\right)^{2-\gamma},
\end{aligned}
$$

for sufficiently large $n$. We therefore have

$$
\frac{u_{m}^{n+1}}{\left(u_{m}^{n}\right)^{2-\gamma}}=\frac{1}{\left(u_{m}^{n}\right)^{1-\gamma}}+2 \frac{\lambda_{n}}{\left(u_{m}^{n}\right)^{1-\gamma}}\left(\frac{u_{m-1}^{n}}{u_{m}^{n}}-1\right)+\tau .
$$

Since $u_{m}^{n} \rightarrow \infty, \lambda_{n} \rightarrow 0$ as $n \rightarrow \infty$, and $0<u_{m-1}^{n} / u_{m}^{n}<1$, the right hand side tends to $\tau$ as $n \rightarrow \infty$. 
We next prove that for $\gamma<1$,

$$
\liminf _{n \rightarrow \infty} \frac{u_{m-1}^{n}}{\left(u_{m}^{n}\right)^{\beta}}>0
$$

where $\beta=(1-\gamma) /(2-\gamma)$. In fact, as we saw above, we have $u_{m-1}^{n+1} \geq \lambda_{n} u_{m}^{n}$. This equation and (9) yield

$$
\frac{u_{m-1}^{n+1}}{\left(u_{m}^{n+1}\right)^{\beta}} \geq \frac{\lambda_{n} u_{m}^{n}}{\left(u_{m}^{n+1}\right)^{\beta}}=\frac{\left(u_{m}^{n}\right)^{1-\gamma}}{\left(u_{m}^{n+1}\right)^{\beta}} \frac{\lambda_{n} u_{m}^{n}}{\left(u_{m}^{n}\right)^{1-\gamma}} \rightarrow \lambda \tau^{-\beta} \quad(\text { as } n \rightarrow \infty),
$$

which implies (10).

We are now in a position to prove (8). By (6), we have for sufficiently large $n$

$$
\begin{aligned}
u_{m-2}^{n+1} & \geq\left(1-2 \lambda_{n}\right) u_{m-2}^{n}+\lambda_{n} u_{m-1}^{n} \\
& =\left(1-2 \lambda_{n}\right) u_{m-2}^{n}+\lambda \frac{u_{m-1}^{n}}{\left(u_{m}^{n}\right)^{\gamma}} \\
& =\left(1-2 \lambda_{n}\right) u_{m-2}^{n}+\lambda \frac{u_{m-1}^{n}}{\left(u_{m}^{n}\right)^{\beta}}\left(u_{m}^{n}\right)^{\frac{\gamma^{2}-3 \gamma+1}{2-\gamma}} .
\end{aligned}
$$

Since $0<\gamma \leq(3-\sqrt{5}) / 2$, the last term of the right hand side is greater than a positive constant, say $M>0$. Since $\lambda_{n} \rightarrow 0$, We have

$$
\liminf _{n \rightarrow \infty} u_{m-2}^{n+1} \geq \liminf _{n \rightarrow \infty} u_{m-2}^{n}+M .
$$

But this is true only if $\liminf _{n \rightarrow \infty} u_{m-2}^{n}=\infty$.

REMARK 3.1. It is a good question to ask whether $\lim _{\sup } \sin _{n \rightarrow \infty} u_{m-2}^{n}<\infty$ in the case of $(3-\sqrt{5}) / 2<\gamma<1$. We can prove this under the assumption that there exists a $k>0$ such that $\lim _{n \rightarrow \infty} u_{m-1}^{n}\left(u_{m}^{n}\right)^{-k}$ exists. Although this assumption seems to be plausible to us, we can not prove $\lim \sup _{n \rightarrow \infty} u_{m-2}^{n}<\infty$ without assuming this. Hence we do not dare to bother the reader by providing a proof of this weak result.

A similar consideration leads us to the following theorem:

Theorem 3.2. For all $0<\gamma \leq(5-\sqrt{17}) / 4$, it holds that

$$
\lim _{n \rightarrow \infty} u_{m-3}^{n}=\infty \text {. }
$$

Proof. We first note that for $\gamma \leq(3-\sqrt{5}) / 2$

$$
\liminf _{n \rightarrow \infty} \frac{u_{m-2}^{n}}{\left(u_{m-1}^{n}\right)^{\frac{\gamma^{2}-3 \gamma+1}{1-\gamma}}}>0 .
$$

In fact, the equation (6) gives us for sufficiently large $n$,

$$
u_{m-2}^{n+1} \geq \lambda_{n} u_{m-1}^{n}=\lambda \frac{u_{m-1}^{n}}{\left(u_{m}^{n}\right)^{\gamma}}=\lambda\left(\frac{u_{m-1}^{n}}{\left(u_{m}^{n}\right)^{\frac{1-\gamma}{2-\gamma}}}\right)^{\frac{\gamma(2-\gamma)}{1-\gamma}}\left(u_{m-1}^{n}\right)^{\frac{\gamma^{2}-3 \gamma+1}{1-\gamma}} .
$$

Therefore, by (10), we have

$$
\liminf _{n \rightarrow \infty} \frac{u_{m-2}^{n}}{\left(u_{m-1}^{n}\right)^{\frac{\gamma^{2}-3 \gamma+1}{1-\gamma}}} \geq \lambda \liminf _{n \rightarrow \infty}\left(\frac{u_{m-1}^{n}}{\left(u_{m}^{n}\right)^{\frac{1-\gamma}{2-\gamma}}}\right)^{\frac{\gamma(2-\gamma)}{1-\gamma}}>0 .
$$


We now come back to (6) to have,

$$
\begin{aligned}
u_{m-3}^{n+1} & \geq\left(1-2 \lambda_{n}\right) u_{m-3}^{n}+\lambda_{n} u_{m-2}^{n}=\left(1-2 \lambda_{n}\right) u_{m-3}^{n}+\lambda \frac{u_{m-2}^{n}}{\left(u_{m}^{n}\right)^{\gamma}} \\
& =\left(1-2 \lambda_{n}\right) u_{m-3}^{n}+\lambda \frac{u_{m-2}^{n}}{\left(u_{m-1}^{n}\right)^{\frac{\gamma^{2}-3 \gamma+1}{1-\gamma}}}\left(\frac{u_{m-1}^{n}}{\left(u_{m}^{n}\right)^{\frac{1-\gamma}{2-\gamma}}}\right)^{\frac{\gamma^{2}-3 \gamma+1}{1-\gamma}}\left(u_{m}^{n}\right)^{\frac{2 \gamma^{2}-5 \gamma+1}{2-\gamma}} .
\end{aligned}
$$

This, (10), and (11) imply the present theorem.

REMARK 3.2. Along the same approach, we can prove that the number of blow-up points increases as $\gamma$ decreases.

3.2. Implicit scheme. We now consider the following scheme.

$$
\frac{u_{j}^{n+1}-u_{j}^{n}}{\triangle t_{n}}=\frac{u_{j+1}^{n+1}-2 u_{j}^{n+1}+u_{j-1}^{n+1}}{h^{2}}+\left(u_{j}^{n}\right)^{2} \quad\left(\begin{array}{l}
j=1, \cdots, N-1 \\
n=0,1, \cdots
\end{array}\right),
$$

where $\Delta t_{n}$ is given by $(5)$.

Theorem 3.3. Let $\left\{u_{j}^{n}\right\}$ be the solution of (12) with (5). Then we have

$$
\begin{array}{ll}
\lim _{n \rightarrow \infty} u_{m-1}^{n}=\infty & (0<\gamma \leq 1), \\
\lim _{n \rightarrow \infty} u_{m-2}^{n}=\infty & (0<\gamma \leq 2 / 3), \\
\lim _{n \rightarrow \infty} u_{m-3}^{n}=\infty & (0<\gamma \leq 1 / 2) .
\end{array}
$$

Proof. We first prepare a lemma.

Lemma 3.1. For all $0<\gamma<1$,

$$
\lim _{n \rightarrow \infty} \frac{u_{m}^{n}}{u_{m}^{n+1}}=0, \quad \lim _{n \rightarrow \infty} \frac{u_{m}^{n+1}}{\left(u_{m}^{n}\right)^{2-\gamma}}=\tau .
$$

Proof. Since

$$
\begin{aligned}
\left(1+2 \lambda_{n}\right) u_{m}^{n+1} & =2 \lambda_{n} u_{m-1}^{n+1}+u_{m}^{n}+\triangle t_{n}\left(u_{m}^{n}\right)^{2} \\
& =2 \lambda_{n} u_{m-1}^{n+1}+\left(1+\tau\left(u_{m}^{n}\right)^{1-\gamma}\right) u_{m}^{n},
\end{aligned}
$$

for sufficiently large $n$, we have

$$
\frac{u_{m}^{n}}{u_{m}^{n+1}}=\frac{1+2 \lambda_{n}}{1+\tau\left(u_{m}^{n}\right)^{1-\gamma}}\left(1-\frac{2 \lambda_{n} u_{m-1}^{n+1}}{\left(1+2 \lambda_{n}\right) u_{m}^{n+1}}\right) .
$$

Since $\lambda_{n} \rightarrow 0$ and $0<u_{m-1}^{n+1}<u_{m}^{n+1}$, the right hand side tends to zero, proving the first equation of the present lemma.

On the other hand, (16) can be written as

$$
1=\frac{2 \lambda_{n} u_{m-1}^{n+1}}{\left(1+2 \lambda_{n}\right) u_{m}^{n+1}}+\frac{u_{m}^{n}}{\left(1+2 \lambda_{n}\right) u_{m}^{n+1}}+\tau \frac{\left(u_{m}^{n}\right)^{2-\gamma}}{\left(1+2 \lambda_{n}\right) u_{m}^{n+1}} .
$$


Since the first two terms of the right hand side tend to zero, one obtains

$$
\lim _{n \rightarrow \infty} \frac{u_{m}^{n+1}}{\left(u_{m}^{n}\right)^{2-\gamma}}=\tau .
$$

The equation (13) is now proved as follows: For sufficiently large $n$,

$$
\left(1+2 \lambda_{n}\right) u_{m-1}^{n+1} \geq \lambda_{n} u_{m}^{n+1} \geq \lambda_{n} u_{m}^{n}=\lambda\left(u_{m}^{n}\right)^{1-\gamma} \rightarrow \infty
$$

which implies (13) in the case of $\gamma<1$. If $\gamma=1,(13)$ is proved in [5].

LEMMA 3.2. It holds that for all $\gamma \leq 1$

$$
\liminf _{n \rightarrow \infty} \frac{u_{m-1}^{n}}{\left(u_{m}^{n}\right)^{\frac{2-2 \gamma}{2-\gamma}}}>0 .
$$

Proof. The equation (12) implies that

$$
\begin{aligned}
u_{m-1}^{n+1} & \geq \frac{\lambda_{n}}{1+2 \lambda_{n}} u_{m}^{n+1}=\frac{\lambda}{1+2 \lambda_{n}} \frac{u_{m}^{n+1}}{\left(u_{m}^{n}\right)^{\gamma}} \\
& =\frac{\lambda}{1+2 \lambda_{n}}\left(\frac{u_{m}^{n+1}}{\left(u_{m}^{n}\right)^{2-\gamma}}\right)^{\frac{\gamma}{2-\gamma}}\left(u_{m}^{n+1}\right)^{\frac{2-2 \gamma}{2-\gamma}} .
\end{aligned}
$$

Then Lemma 3.1 yields the desired result.

We are now ready to prove (14). By (12),

$$
\begin{aligned}
\left(1+2 \lambda_{n}\right) u_{m-2}^{n+1} & \geq u_{m-2}^{n}+\lambda_{n} u_{m-1}^{n+1}=u_{m-2}^{n}+\lambda \frac{u_{m-1}^{n+1}}{\left(u_{m}^{n}\right)^{\gamma}} \\
& =u_{m-2}^{n}+\lambda \frac{u_{m-1}^{n+1}}{\left(u_{m}^{n+1}\right)^{\frac{2-2 \gamma}{2-\gamma}}}\left(\frac{u_{m}^{n+1}}{\left(u_{m}^{n}\right)^{2-\gamma}}\right)^{\frac{2-2 \gamma}{2-\gamma}}\left(u_{m}^{n}\right)^{2-3 \gamma} .
\end{aligned}
$$

Using Lemmas 3.1 and 3.2, we can prove (14) just as in the same way as we proved (8).

We need the following lemma for the proof of (15).

Lemma 3.3. Assume that $0<\gamma \leq \frac{2}{3}$. Then

$$
\liminf _{n \rightarrow \infty} \frac{u_{m-2}^{n}}{\left(u_{m}^{n}\right)^{\frac{2-3 \gamma}{2-\gamma}}}>0 .
$$

Proof. By virtue of (12), we have

$$
\begin{aligned}
\left(1+2 \lambda_{n}\right) u_{m-2}^{n+1} & \geq \lambda_{n} u_{m-1}^{n+1}=\lambda \frac{u_{m-1}^{n+1}}{\left(u_{m}^{n}\right)^{\gamma}} \\
& \geq \lambda \frac{u_{m-1}^{n+1}}{\left(u_{m}^{n+1}\right)^{\frac{2-2 \gamma}{2-\gamma}}}\left(\frac{\left(u_{m}^{n+1}\right)^{\frac{1}{2-\gamma}}}{u_{m}^{n}}\right)^{\gamma}\left(u_{m}^{n+1}\right)^{\frac{2-3 \gamma}{2-\gamma}}
\end{aligned}
$$

which, together with Lemma 3.1 and 3.2, implies the present lemma. 
We now consider (12) with $j=m-3$ :

$$
\begin{aligned}
\left(1+2 \lambda_{n}\right) u_{m-3}^{n+1} & \geq u_{m-3}^{n}+\lambda_{n} u_{m-2}^{n+1}=u_{m-3}^{n}+\lambda \frac{u_{m-2}^{n+1}}{\left(u_{m}^{n}\right)^{\gamma}} \\
& =u_{m-3}^{n}+\lambda \frac{u_{m-2}^{n+1}}{\left(u_{m}^{n+1}\right)^{\frac{2-3 \gamma}{2-\gamma}}}\left(\frac{u_{m}^{n+1}}{\left(u_{m}^{n}\right)^{2-\gamma}}\right)^{\frac{2-3 \gamma}{2-\gamma}}\left(u_{m}^{n}\right)^{2-4 \gamma} .
\end{aligned}
$$

This completes the proof of the theorem.

If this process is repeated, we have the following result. have

Theorem 3.4. Let $k \in\{1,2, \cdots, m-1\}$. Assume that $0<\gamma \leq \frac{2}{k+1}$. Then we

$$
\lim _{n \rightarrow \infty} u_{m-k}^{n}=\infty \quad \text { and } \quad \liminf _{n \rightarrow \infty} \frac{u_{m-k}^{n}}{\left(u_{m}^{n}\right)^{\frac{2-(k+1) \gamma}{2-\gamma}}}>0 .
$$

REMARK 3.3. Since a large $\gamma$ is uneconomical in the sense that $\triangle t_{n}$ becomes too small, and since the number of the numerical blow-up points increases as $\gamma$ decreases, there must be an optimal $\gamma$. We do not have conclusive evidence that $H(s)=s^{\alpha}$ is the best in the case of $f(u)=u^{1+\alpha}$, but, based upon the analysis of the present section and the numerical experiments in [7], we may say at least that this is not a bad choice.

REMARK 3.4. We have

$$
\liminf _{n \rightarrow \infty} \frac{u_{m-1}^{n}}{\left(u_{m}^{n}\right)^{\frac{1-\gamma}{2-\gamma}}}>0 \quad \text { and } \quad \liminf _{n \rightarrow \infty} \frac{u_{m-1}^{n}}{\left(u_{m}^{n}\right)^{\frac{2-2 \gamma}{2-\gamma}}}>0
$$

in the explicit and implicit schemes, respectively. We may therefore say that the implicit scheme speeds up the blow-up for $u_{m-1}^{n}$.

REMARK 3.5. For $\gamma>1$, we may well expect that the numerical solution blows up only at the central point $x_{m}=1 / 2$, but we cannot prove this.

REMARK 3.6. Since the implicit scheme is not quite the same as the explicit one, one may wonder what happens for the Crank-Nicolson scheme:

$\frac{u_{j}^{n+1}-u_{j}^{n}}{\triangle t_{n}}=\frac{1}{2}\left[\frac{u_{j+1}^{n+1}-2 u_{j}^{n+1}+u_{j-1}^{n+1}}{h^{2}}+\frac{u_{j+1}^{n}-2 u_{j}^{n}+u_{j-1}^{n}}{h^{2}}\right]+\frac{1}{2}\left[f\left(u_{j}^{n+1}\right)+f\left(u_{j}^{n}\right)\right]$

This question was asked by Prof. Dongwoo Sheen. Since the Crank-Nicolson scheme is unconditionally stable and of higher order, it is used in many situations and may yield good results in the blow-up problems, too. On the other hand, it is of higher order under the condition that the solution is smooth. In this respect, it may not be very efficient for the blow-up problems. It would be an interesting problem to decide which view is correct. 
4. Blow-up in the case of $f(u)=u[\log (1+u)]^{\beta}$. In this section, we consider the scheme (3) with $f(u)=u[\log (1+u)]^{\beta}$. We assume that $\beta>1$, since all the solutions exist globally in time if $\beta=1$. Problems of this kind were considered by Lacey [12], according to which the blow-up profile varies with $\beta<2, \beta=2,2<\beta$. See section 2. The function $H(s)$ in (4) is chosen as $H(s)=\frac{f(s)}{s}$, which satisfies the assumptions of Theorem 2.1. We therefore consider

$$
\begin{gathered}
\frac{u_{j}^{n+1}-u_{j}^{n}}{\triangle t_{n}}=\frac{u_{j+1}^{n}-2 u_{j}^{n}+u_{j-1}^{n}}{h^{2}}+u_{j}^{n}\left[\log \left(1+u_{j}^{n}\right)\right]^{\beta} \quad\left(\begin{array}{l}
j=1, \cdots, N-1 \\
n=0,1, \cdots
\end{array}\right), \\
\triangle t_{n}=\tau \cdot \min \left\{1, \frac{1}{\left[\log \left(1+u_{m}^{n}\right)\right]^{\beta}}\right\} .
\end{gathered}
$$

For this choice of $H(s)$, we have the following theorem [7].

TheOrem 4.1. Let $\left\{u_{j}^{n}\right\}$ be the solution of (17) and (18). Suppose that the initial data is large so that the solution blows up in finite time. Then, for $\beta>1$,

$$
\begin{array}{cl}
\lim _{n \rightarrow \infty} u_{j}^{n}=\infty, & (j=1, \cdots, m), \\
& \lim _{n \rightarrow \infty} \frac{u_{m}^{n+1}}{u_{m}^{n}}=1+\tau, \\
\lim _{n \rightarrow \infty} \frac{\log \left(1+u_{m}^{n}\right)}{\log \left(1+u_{j}^{n}\right)}=1, & \lim _{n \rightarrow \infty} \frac{\log \left(1+u_{j}^{n+1}\right)}{\log \left(1+u_{j}^{n}\right)}=1, \quad(j=1, \cdots, m) .
\end{array}
$$

According to Theorem 4.1, all the grid points are in the numerical blow-up set independently of $\beta$. This makes a sharp contrast with Lacey's result, which guarantees a single-point blow-up for $\beta>2$. At first glance, there seems to be no ways to distinguish the total and single-point blow-up in the present case of nonlinearity. But from the numerical experiments, we found a rather interesting result: Although the blow-up sets of the numerical solution and the solution for the PDE are different, the graphs of normalized functions seem to confirm Lacey's theorem.
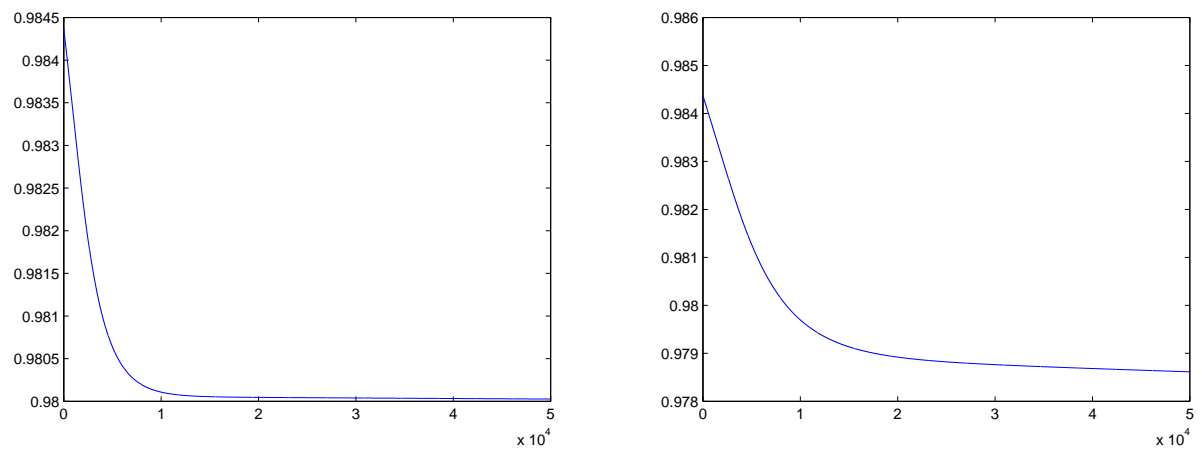

FIG. 1. Left: $\beta=6 / 5$. Right: $\beta=3 / 2$. The abscissa represents $n$. 

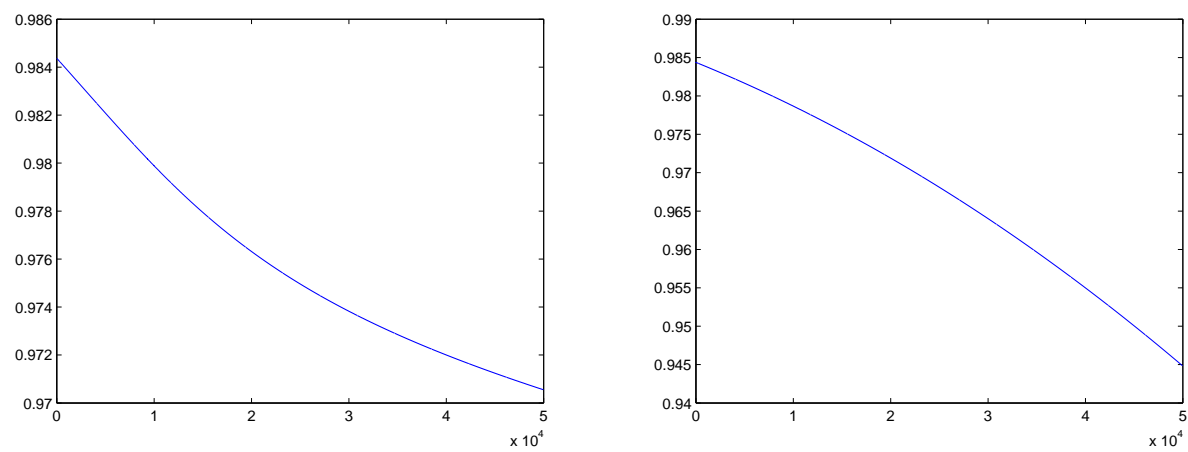

FIG. 2. Left: $\beta=2$. Right: $\beta=3$.
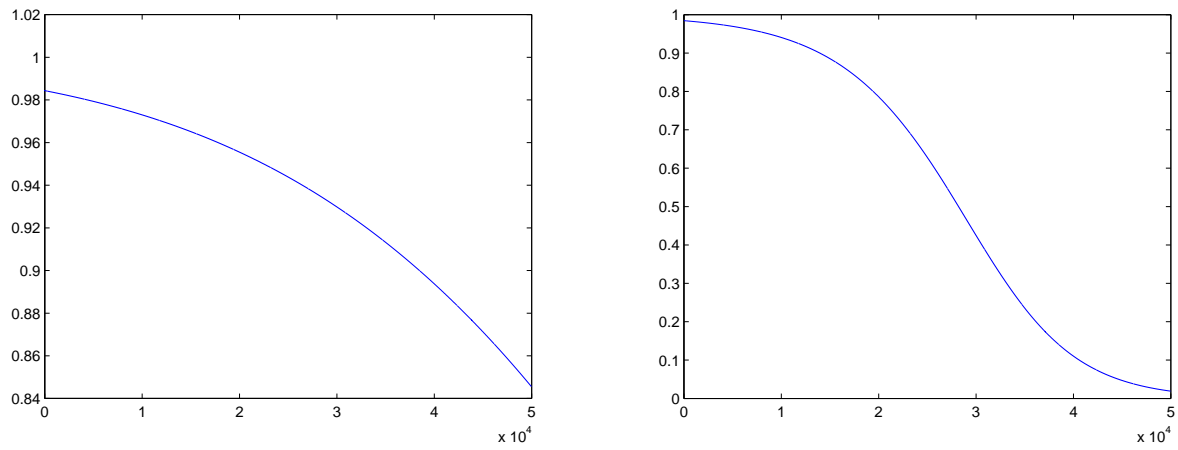

FIG. 3. Left: $\beta=5$. Right: $\beta=10$.

To be more precise, let the initial function is chosen as $100000 x(1-x) \quad(0<$ $x<1)$. The number of grid points and $\tau$ are given as $N=1 / h=16, \tau=0.0001$, respectively. (Since the blow-up in the case of the present nonlinearity is quite slow, we chose a large initial data in order to see the blow-up profile in short time. If $N$ is large, it takes intolerably long time to reach a near-blow-up state. Hence we are forced to choose a small $N$.) Figure 1 shows the graphs of $u_{m-1}^{n} / u_{m}^{n}$ in the cases of $\beta<2$. They suggest that

$$
\liminf _{n \rightarrow \infty} \frac{u_{m-1}^{n}}{u_{m}^{n}}>0 .
$$

On the other hand, Figure 2 and 3, which show the cases where $\beta>2$, seem to suggest that

$$
\lim _{n \rightarrow \infty} \frac{u_{m-1}^{n}}{u_{m}^{n}}=0 .
$$

If this is true, then we may say that Lacey's theorem can be partly realized by the finite difference method. Right now, we are unable to prove the two limit relations above. We instead prove the following related equation.

THEOREM 4.2. Let $\left\{u_{j}^{n}\right\}$ be the solution of (17) and (18). Suppose that the initial data is large so that the solution blows up in finite time. Assumptions (A0)-(A3) 
are assumed. Then, for any $\beta>1$,

$$
\lim _{n \rightarrow \infty} \frac{\lambda_{n} u_{m}^{n}}{u_{m-1}^{n}}=0
$$

This theorem is interpreted as follows. Note that

$$
\lambda_{n}=\frac{\lambda}{\left[\log \left(1+u_{m}^{n}\right)\right]^{\beta}}
$$

tends to zero very slowly. Therefore, even if $u_{m-1}^{n} / u_{m}^{n}$ tends to zero, the convergence is quite slow.

Proof of the Theorem. Note first that

$$
\lim _{n \rightarrow \infty} \frac{\lambda_{n+1} u_{m}^{n+1}}{\lambda_{n} u_{m}^{n}}=1+\tau
$$

which can be derived from Theorem 4.1 and (18). Note next that

$$
\frac{u_{m-1}^{n+1}}{u_{m-1}^{n}}-\frac{\lambda_{n} u_{m}^{n}}{u_{m-1}^{n}}=1-2 \lambda_{n}+\triangle t_{n}\left[\log \left(1+u_{m-1}^{n}\right)\right]^{\beta}+\frac{\lambda_{n} u_{m-2}^{n}}{u_{m-1}^{n}},
$$

from which follows

$$
\lim _{n \rightarrow \infty}\left(\frac{u_{m-1}^{n+1}}{u_{m-1}^{n}}-\frac{\lambda_{n} u_{m}^{n}}{u_{m-1}^{n}}\right)=1+\tau
$$

Thus,

$$
\begin{aligned}
\frac{\lambda_{n+1} u_{m}^{n+1}}{u_{m-1}^{n+1}} & =\frac{\lambda_{n+1} u_{m}^{n+1}}{\lambda_{n} u_{m}^{n}} \frac{u_{m-1}^{n}}{u_{m-1}^{n+1}} \frac{\lambda_{n} u_{m}^{n}}{u_{m-1}^{n}} \\
& =\frac{\frac{\lambda_{n+1} u_{m}^{n+1}}{\lambda_{n} u_{m}^{n}}}{\frac{u_{m-1}^{n+1}}{u_{m-1}^{n}}-\frac{\lambda_{n} u_{m}^{n}}{u_{m-1}^{n}}+\frac{\lambda_{n} u_{m}^{n}}{u_{m-1}^{n}}} \cdot \frac{\lambda_{n} u_{m}^{n}}{u_{m-1}^{n}}
\end{aligned}
$$

If we set $U^{n}=\lambda_{n} u_{m}^{n} / u_{m-1}^{n}$, then this equation can be written as

$$
U^{n+1}=\frac{w^{n}}{v^{n}+U^{n}} U^{n}
$$

with $\lim _{n \rightarrow \infty} w_{n}=\lim _{n \rightarrow \infty} v_{n}=1+\tau$.

For any $\epsilon>0$, there exists an $n_{0}$ such that

$$
w_{n}<1+\tau+\epsilon, \quad v_{n}>1+\tau-\epsilon
$$

for all $n \geq n_{0}$. We then have

$$
U^{n+1} \leq \frac{1+\tau+\epsilon}{1+\tau-\epsilon+U^{n}} U^{n}
$$

for all $n \geq n_{0}$. Let $\tilde{U}^{n}$ be defined by $\tilde{U}^{n_{0}}=U^{n_{0}}$ and

$$
\tilde{U}^{n+1}=\frac{1+\tau+\epsilon}{1+\tau-\epsilon+\tilde{U}^{n}} \tilde{U}^{n} \quad\left(n \geq n_{0}\right) .
$$


It is not difficult to prove that $U^{n} \leq \tilde{U}^{n}$ for all $n \geq n_{0}$ and that $\lim _{n \rightarrow \infty} \tilde{U}^{n}=2 \epsilon$. Since $\epsilon$ is arbitrary, it follows that $\lim _{n \rightarrow \infty} U^{n}=0$.

Corollary 4.1.

$$
\lim _{n \rightarrow \infty} \frac{u_{m-1}^{n+1}}{u_{m-1}^{n}}=1+\tau
$$

Proof. This follows from the present theorem and (22).

If we employ the same method, we have the following

Theorem 4.3. For $\beta>1$,

$\lim _{n \rightarrow \infty} \frac{u_{j}^{n+1}}{u_{j}^{n}}=1+\tau \quad(j=1,2, \cdots, m), \quad \lim _{n \rightarrow \infty} \lambda_{n} \frac{u_{j+1}^{n}}{u_{j}^{n}}=0 \quad(j=1,2, \cdots, m-1)$.

\section{REFERENCES}

[1] L. Abia, J.C. López-Marcos, And J. Martínez, Blow-up for semidiscretizations of reactiondiffusion equations, Appl. Numer. Math., 20 (1996), pp. 145-156.

[2] L. Abia, J.C. López-Marcos, and J. Martínez, On the blow-up time convergence of semidiscretizations of reaction-diffusion equations, ibid., 26 (1998), pp. 399-414.

[3] L.M. Abia, J.C. López-Marcos, And J. Martínez, The Euler method in the numerical integration of reaction-diffusion problems with blow-up, ibid., 38 (2001), pp. 287-313.

[4] L.A. Caffarrelli and A. Friedman, Blow-up of solutions of nonlinear heat equations, J. Math. Anal. Appl., 129 (1988), pp. 409-419.

[5] Y.-G. Chen, Asymptotic behaviours of bolwing-up solutions for finite difference analogue of $u_{t}=u_{x x}+u^{1+\alpha}$, J. Fac. Sci. Univ. Tokyo Sect. IA, 33 (1986), pp. 541-474.

[6] C.-H. Сho, On a finite difference scheme for the parabolic blow-up problems, Master's Thesis, Research Institute for Mathematical Sciences, Kyoto university (2005).

[7] C.-H. Сho, S. Hamada, and H. Окамото, On the finite difference approximation for a parabolic blow-up problem, Japan J. Indust. Appl. Math., 24 (2007), pp. 105-134.

[8] K. Deng And H.A. Levine, The role of critical exponents in blow-up theorems: the sequel, J. Math. Anal. Appl., 243 (2000), pp. 85-126.

[9] M. Fila And H. Matano, Blow-up in nonlinear heat equations from the dynamical systems point of view, in "Handbook of Dynamical Systems", ed. B. Fiedler, Elsevier (2002), pp. $723-758$.

[10] A. Friedman And B. McLeod, Blow-up of positive solutions of semilinear heat equations, Indiana Univ. Math. J., 34 (1985), pp. 425-447.

[11] S. Iто, On blow-up of positive solutions of semilinear parabolic equations, J. Fac. Sci. Univ. Tokyo, Sect. IA, 37 (1990), pp. 527-536.

[12] A.A. LACEY, Global blow-up of a nonlinear heat equation, Proc. R. Soc. Edin., 104 A (1986), pp. 161-167.

[13] T. Nakagawa, Blowing up of a finite difference solution to $u_{t}=u_{x x}+u^{2}$, Appl. Math. Optim., 2 (1976), pp. 337-350.

[14] F. Weissler, Single point blowup of semilinear initial value problems, J. Diff. Eqns., 55 (1984), pp. 202-224.

[15] E. YANAGidA, Blow-up of solutions of nonlinear heat equations, in "Blow-up and Condensation", ed. E. Yanagida, University of Tokyo Press (2006), pp. 1-50, (in Japanese). 
